\title{
Reusable Frame for Pedicle Protection in Heel and Ankle Defects
}

\author{
${ }^{1}$ Sonal T Lal, ${ }^{2}$ Chandanpreet K Kahlon, ${ }^{3}$ Deepak J Bhatti
}

\begin{abstract}
Introduction: It has always been a challenging task for the reconstructive surgeons to resurface the lost skin and soft tissue over the heel. This is done by use of skin grafts, local flap or microvascular free flap (MFF). The postoperative care and splintage of these limbs is a difficult task, and often the success or failure of the flap will depend on proper postoperative splintage and positioning of the operated limb. A simple and reusable frame is being described for pedicle protection in flap surgery for posterior heel and ankle wounds.
\end{abstract}

Aims and objective: To devise a reusable frame for pedicle protection in heel and ankle defects.

Materials and methods: A reusable frame splint designed by holding up the foot against gravity at a narrower circumference of forefoot just proximal to the wider circumference of the foot at the metatar sophalangeal joint (MTP) level. This frame was used in the early postoperative period for positioning the lower limb in patients undergoing surgery for coverage of posterior heel defects.

Result: A total of 14 patients underwent wound coverage of post heel defect over a period of 3 years. Out of these 14 patients, nine had reverse sural artery pedicle flap, three had splitthickness skin grafting and remaining two had MFF done. In all the cases, a specially designed frame was used for pedicle protection. One patient had developed erosion and blistering, which healed subsequently.

Conclusion: This innovation in the splint helps in providing adequate elevation of limb and protection of pedicle of local or MFF used for coverage of heel and ankle defects.

Keywords: Heel defects, Metatarsophalangeal joints, Pedicle protection, Reusable frame splint.

How to cite this article: Lal ST, Kahlon CK, Bhatti DJ. Reusable Frame for Pedicle Protection in Heel and Ankle Defects. J Foot Ankle Surg (Asia Pacific) 2018;5(2):56-58.

Source of support: Nil

Conflict of interest: None

\footnotetext{
${ }^{1,2}$ Associate Professor, ${ }^{3}$ Professor

1,3Department of Plastic Surgery, Guru Gobind Singh Medical College, Faridkot, Punjab, India

${ }^{2}$ Department of Physical and Medical Rehabilitation, Guru Gobind Singh Medical College, Faridkot, Punjab, India

Corresponding Author: Sonal T Lal, Associate Professor, Department of Plastic Surgery, Guru Gobind Singh Medical College, Faridkot, Punjab, India, e-mail: sonalbanipal@gmail.com
}

\section{INTRODUCTION}

Wounds over the heel need coverage with skin grafts, local flap or free flaps. The need for protection of the graft or flap used for coverage of the heel defect in early postoperative period is most important for the success of the surgery. The objective of this study is to introduce a reusable frame/splint that can provide adequate elevation and hold-up the foot against gravity for protection of the pedicle of the flap in wound coverage surgery for posterior heel defects. The principle of this splint is based on gripping the foot at a narrower circumference proximally so that the distal wider portion prevents the foot from slipping down.

\section{MATERIALS AND METHODS}

A total of 14 patients were included in the study done over a period of three years in the department of plastic surgery at our institution. These patients underwent wound coverage over the posterior heel defects. In nine patients reverse sural artery flap was done and in three of these patients, the flaps were delayed and insetting done after a week. In three cases, wound coverage was done by use of split-thickness skin graft and in the remaining two cases MFF was done for resurfacing the skin and soft tissue defects of the heel. In all cases, a specially designed reusable frame was used in the early postoperative period, for protection of graft or flap.

\section{Principle and Design}

The frame was designed with an idea of suspension of the lower limb by gripping the foot at the narrowest area proximally so that the distal wider area prevents the foot from slipping down.

Measurements of the circumference of the foot at different levels among 20 males and 20 females in the age group of 20 to 60 years were taken. The circumference over the knuckles corresponding to the MTP joint level (Fig. 1) and the area proximal to the MTP joints corresponding to the body of metatarsals in the forefoot (Fig. 1) was measured. It was noted that the foot circumference is slightly less over a portion on forefoot just proximal to the MTP joint compared to the MTP joint level and subsequently the circumference increases over the midfoot level. A ratio of the circumference at the MTP joint level to a portion proximal to MTP joint among 


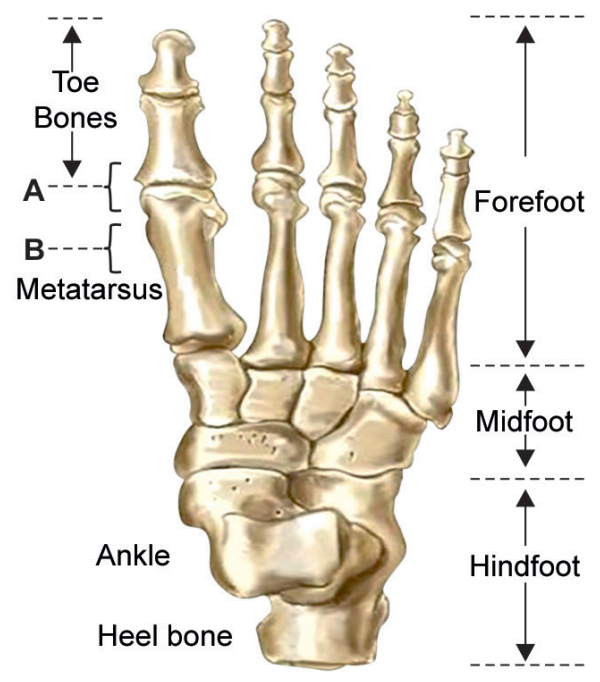

Fig. 1: Splint paper

males was found to be 1.2 and among females was 1.1. Working on these findings a reusable frame/splint was devised which grips the forefoot at a level just proximal to the level of MTP joints thus preventing it from slipping down. This helps in keeping, the heel and the pedicle region free.

The splint was fabricated at a local metal workshop using steel bars, foam sheet, polyvinyl chloride (PVC) sheet and velcro, with inputs from an experienced orthotist. After initial trials, the final prototype was made using stainless steel to aid easy disinfection between repeated uses. The frame is made in a shape of a rightangled triangle (Fig. 2A). The two right angle triangles are joined by two steel bars at the foot end and one steel bar at the proximal end. The short vertical limb of the triangle is 12 inches long, the horizontal limb is 22 inches long, and the hypotenuse is 25 inches long. The horizontal steel bars joining the two triangles, two at the distal end and one at the proximal end are nine inches long. A foam sheet supported by $1 / 8$ th inch thick PVC sheet in a dimension of $2.5 \times 7$ inches at the distal end attached to the upper steel bar joining the two triangles. Second foam

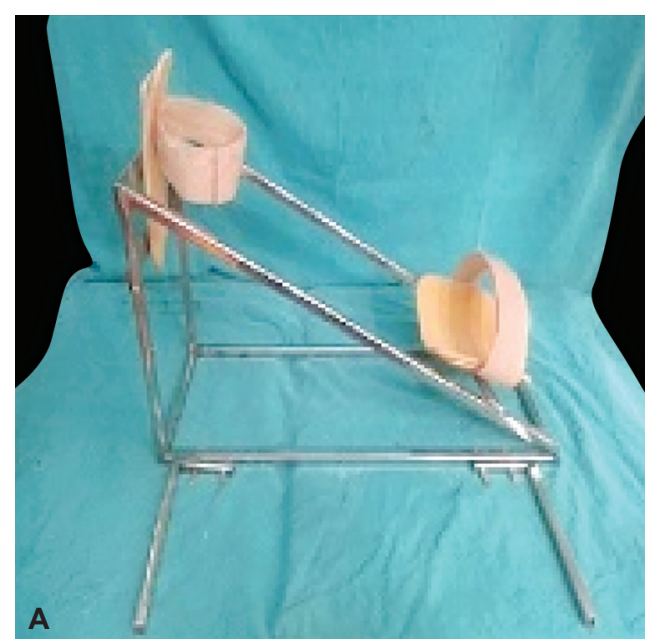

sheet supported by PVC sheet $6 \times 6$ inches is attached to a steel bar fixed 5 inches from the proximal end of the splint attached on the hypotenuse limbs. Third removable foam sheet supported by PVC sheet $8 \times 12$ inches in dimension with attached Velcro was used to support the middle third of the leg when the final insetting of the flap was done. It was not used in cases of flap delay (Fig. 2B). Two right-angled steel bars measuring $3 \times 8$ inches with two holes for screw and nut fixation on to the horizontal limb of the right-angled triangle on both sides are made. These angled bars are attached on the ipsilateral side of the splinted limb to provide lateral support and prevent the splint from rolling at the side. After dressing the limb the foot is placed on the distal foam sheet and the Velcro is tightened over the forefoot just proximal to the MTP joint level (Figs 3 and 4). The calf region lies over the proximal foam sheet and the Velcro is tightened there. A pillow is placed under the thigh to prevent hyperextension of the knee joint. This serves to stabilize the ankle joint, keeps the heel region free of any compression and elevates the limb by 30 degrees. It also allows for easier inspection and convenient dressing change over the flap.

\section{RESULTS}

In all the 14 patients under study, the graft and the flaps were well protected by the use of the splint. Frequent monitoring of the flaps could be comfortably done. Bedside dressing changes were easily done. One patient developed erosions and blistering due to the pressure of the foot strap, which healed subsequently.

\section{DISCUSSION}

Resurfacing of wounds over heel has always been a challenging task. The various surgical options for the wound coverage of heel defect include skin grafting, local flaps, and MFF. The success of the flap surgeries often depends on proper postoperative positioning and splintage of the operated limb.

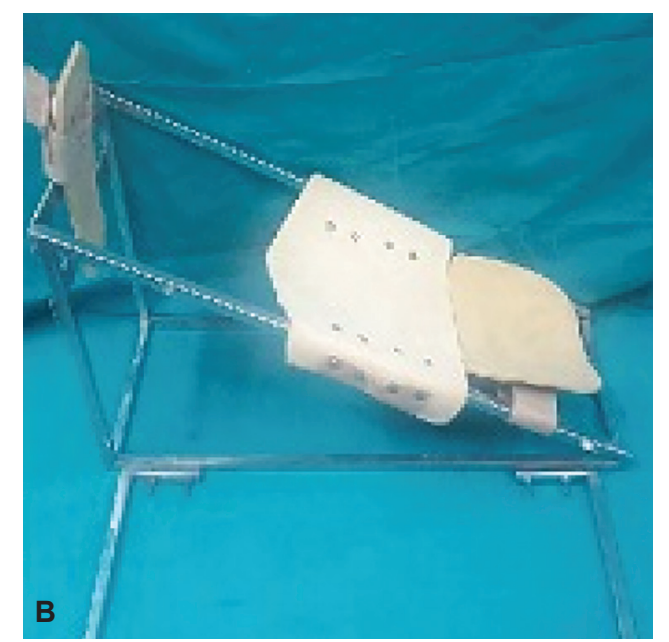

Figs 2A and B: Splint paper 

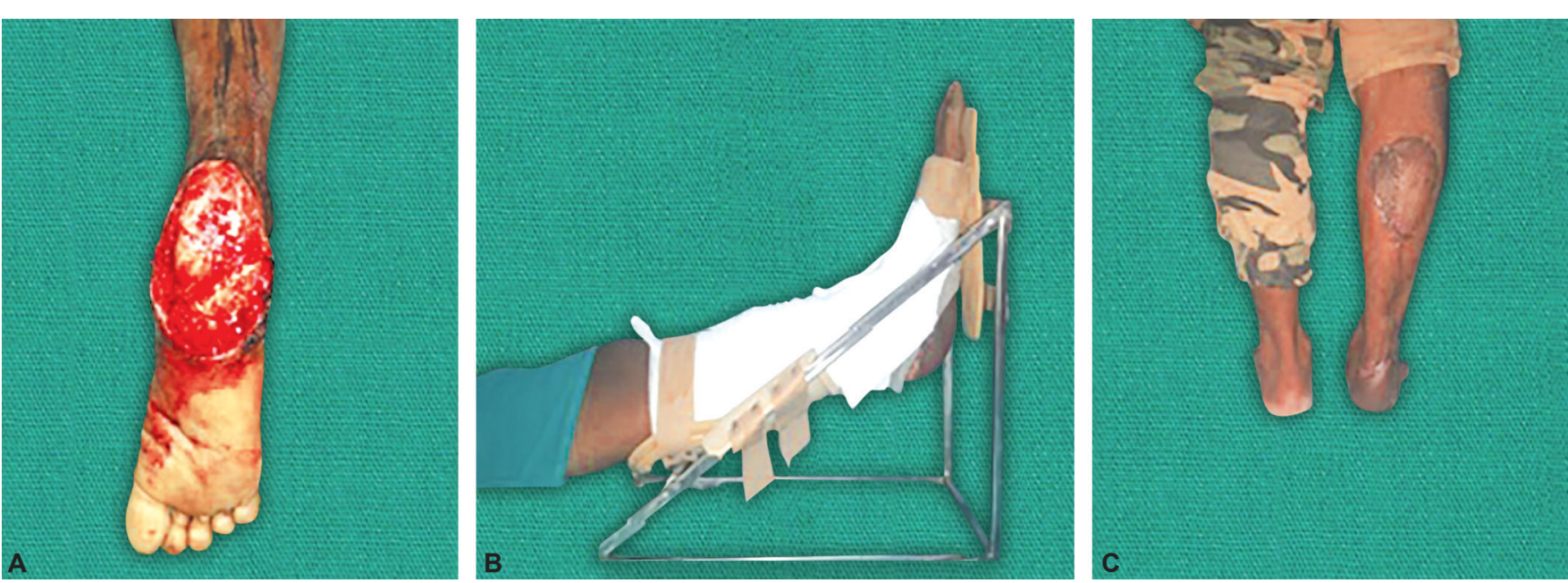

Figs 3A to C: Splint Paper. (A) Posterior heel defect; (B) Operated limb positioned on the splint after sural artery flap insetting (note: the use of third foam sheet); (C) At 3 months of follow-up
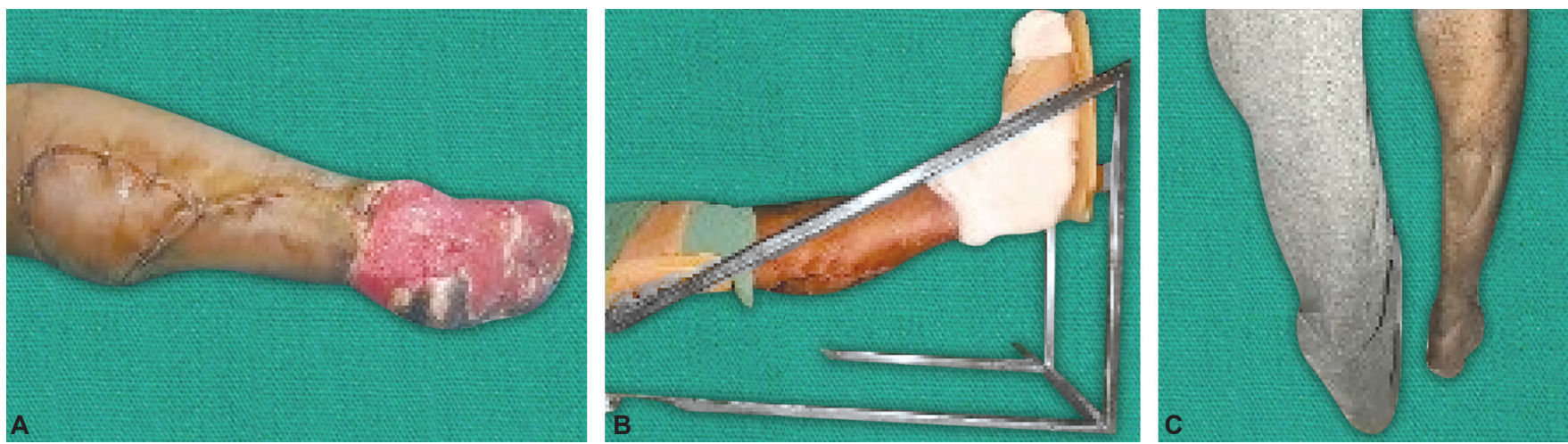

Figs 4A to C: Splint Paper. (A) Degloving injury foot; (B) Operated limb positioned on the splint after sural at Lery flap delay surgery; (C) At 1 months of follow-up

An ideal splint should immobilize and elevate the limb without being constricting or compressive. It should be able to allow for frequent flap inspections and amenable to convenient dressing changes bedside protecting the limb from sudden posture change and falling down. ${ }^{1}$ Conventional methods of using bulky dressings with plaster of paris are unpredictable in protecting the graft or the flap. Moreover, monitoring the flap would be difficult, and it becomes cumbersome for wound care. ${ }^{2}$ Alternative methods like keeping the patient in a prone position are very uncomfortable for the patient over the prolonged period. Invasive methods like external fixators ${ }^{3}$ and ring fixators ${ }^{2}$ have been used. For postoperative limb splintage, other methods like Bohler Braun splint ${ }^{4}$ and Thomas splint ${ }^{5}$ have been used. There are reports of some innovative splint design like the modified pillow splint. ${ }^{6}$ The Lucknow splint used the Kramer wire for making the of floading slab after flaps to the heel. ${ }^{1}$ Another splint, based on the similar idea as of the Lucknow splint, using plaster of paris and saline bottle has been used.

Our innovative reusable frame is simple and comfortable for patients. It is a one-time investment and can be used repeatedly. It can be used for protection of pedicle of local flaps, in cases of flap delay and MFF used for resurfacing of the lost soft tissue of the heel and ankle area. It is recommended to use adequate padding under the foot strap.

\section{CONCLUSION}

This innovative reusable frame has been found useful in managing the heel and ankle defect.

\section{REFERENCES}

1. Upadhyaya DN, Khanna V, Pandey A, Puri A, Kohli R. The Lucknow splint. Indian J Plast Surg 2012;45:587-588.

2. Kamath JB. A simple method for pedicle protection in flap surgery for posterior heel defects. Indian J Plast Surg 2003;36:104-105.

3. Gregory A, Buford MD, Mark A. A novel method for LowerExtremity Immobilization after free flap reconstruction of posterior heel defects. Plast Reconstr Surg 2003;111:821.

4. Ninan S, Manigandan C, Gupta AK. Post operative care of flaps using the Bohler braun frame. An innovation. Plast Reconstr Surg 2005;115:676-677.

5. Bhaskara KG, Kale SM. Use of Thomas splint in salvaging free flaps of lower limb in violent postoperative patients. Indian J Plast Surg 2009;42:271-272.

6. Ellur S. Modified pillow splint. Indian J Plast Surg 2011; 44:529-530.

7. Venkatramani H, Jain DA, Sabapathy SR. A useful modification of plaster backslab to off-load pressure from reconstructions of the heel and elbow. Indian J Plast Surg 2012;45: 588-589. 